\title{
Early Myoclonic Encephalopathy
}

National Cancer Institute

\section{Source}

National Cancer Institute. Early Myoclonic Encephalopathy. NCI Thesaurus. Code

C116593.

A neurologic disorder characterized by frequently recurring myoclonic seizures and other seizure types presenting within the first months of life. 